\title{
Fast Space Mapping with Variable Weight Coefficients for Microwave Device Modeling
}

\author{
Slawomir Koziel, Senior Member, IEEE, and John W. Bandler, Life Fellow, IEEE
}

School of Science and Engineering, Reykjavik University, Kringlunni 1, IS-103 Reykjavik, Iceland Department of Electrical and Computer Eng., McMaster University, Hamilton, ON, Canada L8S 4K1

\begin{abstract}
We describe an improvement of a recent space mapping (SM) modeling approach that uses variable weight coefficients (SM-VWC). Our modification alleviates the main drawback of SM-VWC: the computational overhead related to a separate parameter extraction required for each evaluation of the surrogate model. In our new procedure, the output SM parameters of the surrogate model are obtained by solving a regression problem instead of being determined in the parameter extraction process. This dramatically reduces the evaluation time of the surrogate model. Moreover, the modeling accuracy of the modified technique is even better than the accuracy of the original SM-VWC approach. Examples demonstrate the robustness of our approach.
\end{abstract}

Index Terms-Computer-aided design (CAD), EM modeling, space mapping, surrogate modeling.

\section{INTRODUCTION}

Accurate and computationally efficient models of microwave components and devices are crucial in many areas such as signal processing, wireless communications and biomedical engineering. Full-wave EM simulations of microwave structures offer high accuracy at the cost of CPU effort, which is undesirable from the point of view of direct statistical analysis and design.

The space mapping (SM) [1]-[3] and neuro-spacemapping concept [3]-[6] address this issue by replacing the CPU-intensive EM-simulation-based "fine" model with a computationally cheap surrogate model. The surrogate model is constructed by composing a fast "coarse" model-usually a circuit equivalent of a microwave structure - with suitable auxiliary mappings.

The standard SM modeling methodology [5] provides reasonable accuracy while using a small amount of fine model data. Increasing the amount of fine model data has, however, little or no effect on the accuracy due to parameter extraction being independent of the evaluation point of the surrogate [7].

SM modeling with variable weight coefficients (SMVWC) [7] provides better accuracy, but at the expense of the computational overhead related to a separate parameter extraction required for each evaluation of the surrogate. This limits potential applications of the method.

In this paper, we present a modification of the SMVWC approach, where some of the space mapping parameters are computed by solving a linear regression problem using a set of simple analytical formulas. A regression has a local character (focused on a current evaluation point of the surrogate model) by exploiting the variable-dependent weight factors as in [7]. Therefore, a separate parameter extraction process for each evaluation of the surrogate model is avoided. We demonstrate that the modeling accuracy of the new technique is better than the accuracy of the original SM-VWC approach with substantially lower computational cost.

\section{STANDARD Space MaPPING MODELING. SPaCE MAPPING WITH VARIABLE WEIGHT COEFFICIENTS}

Let $R_{f}: X_{f} \rightarrow R^{m}, X_{f} \subseteq R^{n}$, and $R_{c}: X_{c} \rightarrow R^{m}, X_{c} \subseteq R^{n}$, denote the fine and coarse model response vectors. Let $X_{R} \subseteq X_{f}$ be region of interest where we want an enhanced matching between the surrogate and the fine model. Here, $X_{R}$ is an $n$-dimensional interval in $R^{n}$ with center at reference point $x^{0}=\left[x_{0.1} \ldots x_{0 . n}\right]^{T} \in R^{n}$ and size $\boldsymbol{\delta}=\left[\begin{array}{lll}\delta_{1} & \ldots & \delta_{n}\end{array}\right]^{T}[5]$. Let $X_{B}=\left\{x^{1}, x^{2}, \ldots, x^{N}\right\} \subset X_{R}$ be the base set, such that the fine model response is known at all points $\boldsymbol{x}^{j}, j=1,2, \ldots, N$. Let $\overline{\boldsymbol{R}}_{s}: X_{R} \times X_{p} \rightarrow R^{m}$ be a generic SM surrogate model where $X_{p}$ is a parameter domain. For any given base set $X_{B}$ the standard surrogate model $R_{s . S M}$ is defined as

$$
R_{s, S M}(x)=\bar{R}_{s}(x, \bar{p})
$$

where

$$
\bar{p}=\arg \min _{p \in X_{p}} \sum_{k=1}^{N}\left\|R_{f}\left(x^{k}\right)-\bar{R}_{s}\left(x^{k}, p\right)\right\|
$$

A variety of SM surrogate models is available [1]-[4]. The model often used in practice (e.g., [5]) takes the form of $\bar{R}_{s}(x, p)=\bar{R}_{s}(x, A, B, c)=A \cdot R_{c}(B \cdot x+c)$ and employs both input and output SM. Note that the parameter extraction process (2) is independent of the evaluation point $x$ of the surrogate model. This is the primary reason for which the modeling accuracy of the model (1)-(2) is barely dependent on the number of the base points $N$.

The space mapping with variable weight coefficients (SM-VWC) [7] uses the parameter extraction defined as

$$
\bar{p}=\arg \min _{p \in X_{p}} \sum_{k=1}^{N} w_{k}(\boldsymbol{x})\left\|\boldsymbol{R}_{f}\left(\boldsymbol{x}^{k}\right)-\overline{\boldsymbol{R}}_{s}\left(\boldsymbol{x}^{k}, \boldsymbol{p}\right)\right\|
$$

where 


$$
w_{k}=w_{k}(\boldsymbol{x} ; c, \lambda)=\frac{\exp \left(-\frac{\left\|\boldsymbol{x}-\boldsymbol{x}^{k}\right\|^{2}}{c \cdot \lambda^{2}}\right)}{\sum_{j=1}^{N} \exp \left(-\frac{\left\|\boldsymbol{x}-\boldsymbol{x}^{j}\right\|^{2}}{c \cdot \lambda^{2}}\right)} \quad k=1,2, \ldots, N
$$

with $c>0$ being a control parameter, usually set to 1 , and $\lambda$ being a characteristic distance of the base set defined as

$$
\lambda=\lambda(\boldsymbol{\delta}, N)=\frac{2}{n N^{1 / n}} \sum_{i=1}^{n} \delta_{i}
$$

The SM-VWC model, $R_{s . S M-L W C}$, is defined as in (1), with the parameter extraction (2) replaced by (3)-(5).

It has been demonstrated that SM-VWC gives better accuracy than the standard SM model, and the modeling error can be made as small as required if the base set is sufficiently dense [7]. The drawback of SM-VWC is that each evaluation of the surrogate model (1), (3)-(5) requires a separate parameter extraction, which results in a substantial computational overhead, particularly when a coarse model is evaluated using a circuit simulator.

\section{QUICK SM-VWC MODELING}

The computational complexity of the SM-VWC method can be substantially reduced if the $\boldsymbol{x}$-dependent parameter extraction (3) is replaced by a standard parameter extraction process (2) performed for some of the SM parameters and analytical calculation of the remaining parameters using the variable weight coefficient principle.

Let us consider the following generic SM model:

$$
\bar{R}_{s}(\boldsymbol{x}, \boldsymbol{p})=\overline{\boldsymbol{R}}_{s}(\boldsymbol{x}, A, B, c, d)=A \cdot \boldsymbol{R}_{c}(B \cdot \boldsymbol{x}+\boldsymbol{c})+\boldsymbol{d}
$$

where $A=\operatorname{diag}\left\{a_{1}, \ldots, a_{m}\right\}, B$ is $n \times n$ matrix, $c$ is $n \times 1$ vector and $\boldsymbol{d}=\left[\begin{array}{llll}d_{1} & d_{2} & \ldots & d_{m}\end{array}\right]^{T}$. The model (6) can be enhanced using the frequency SM and/or implicit SM [5].

Parameters $B$ and $c$ are extracted in the $x$-independent process as follows

$$
(B, c)=\arg \min _{B, c} \sum_{k=1}^{N}\left\|R_{f}\left(x^{k}\right)-R_{c}\left(B \cdot x^{k}+c\right)\right\|
$$

For the sake of convenience, we shall use the notation $R_{S . I S M}(x)=R_{c}(B x+c)$, where $B$ and $c$ are obtained by (7).

Parameters $A$ and $\boldsymbol{d}$ are found by solving a linear regression problem

$$
\min _{\boldsymbol{A}, \boldsymbol{d}} \sum_{k=1}^{N} w_{k}(\boldsymbol{x})\left\|\boldsymbol{R}_{f}\left(\boldsymbol{x}^{k}\right)-\left(\boldsymbol{A} \cdot \boldsymbol{R}_{s, L S M}\left(\boldsymbol{x}^{k}\right)+\boldsymbol{d}\right)\right\|^{2}
$$

with weighting coefficients $w_{k}$ determined by (4).

Let us introduce the following notation: $R_{f}(\boldsymbol{x})=\left[R_{f .1}(\boldsymbol{x}) R_{f .2}(\boldsymbol{x}) \ldots R_{f, m}(\boldsymbol{x})\right]^{T}$, where $R_{f, j}(\boldsymbol{x})$ is the $j$ th component of the vector $R_{f}(x)$. Similarly, we will use the notation $R_{\text {c.ISMM }}(\boldsymbol{x})=\left[R_{\text {c.ISMM.1 }}(\boldsymbol{x}) R_{\text {c.ISMM. }}(\boldsymbol{x}) \ldots R_{\text {c.ISM } . m}(\boldsymbol{x})\right]^{T}$.

Problem (8) is then equivalent to $m$ regression problems

$$
\min _{a_{j}, d_{j}} \sum_{k=1}^{N} w_{k}(\boldsymbol{x})\left|R_{f, j}\left(\boldsymbol{x}^{k}\right)-\left(a_{j} \cdot R_{s . L S M . j}\left(\boldsymbol{x}^{k}\right)+d_{j}\right)\right|^{2}
$$

where $j=1, \ldots, m$. The problem (9) can be formulated as

$$
\min _{z_{j}}\left\|Q_{j} z_{j}-Y_{j}\right\|
$$

where

$$
\begin{aligned}
& Q_{j}=\left[\begin{array}{cc}
\sqrt{w_{1}(\boldsymbol{x})} R_{\text {s.LSM }, j}\left(\boldsymbol{x}^{1}\right) & \sqrt{w_{1}(\boldsymbol{x})} \\
\sqrt{w_{2}(\boldsymbol{x})} R_{s, L M, . j}\left(\boldsymbol{x}^{2}\right) & \sqrt{w_{2}(\boldsymbol{x})} \\
\vdots & \vdots \\
\sqrt{w_{N}(\boldsymbol{x})} R_{s, L S M, j}\left(\boldsymbol{x}^{N}\right) & \sqrt{w_{N}(\boldsymbol{x})}
\end{array}\right] \\
& Y_{j}=\left[\begin{array}{lll}
\sqrt{w_{1}(\boldsymbol{x})} R_{s, I S M, j}\left(\boldsymbol{x}^{1}\right) & \cdots & \sqrt{w_{N}(\boldsymbol{x})} R_{s . I S M, j}\left(\boldsymbol{x}^{N}\right)
\end{array}\right]^{T}
\end{aligned}
$$

and

$$
\boldsymbol{z}_{j}=\left[\begin{array}{ll}
a_{j} & d_{j}
\end{array}\right]^{T}
$$

The least-square solution to (10) is given by

$$
\boldsymbol{z}_{j}=\left(Q_{j}^{T} Q_{j}\right)^{-1} Q_{j}^{T} Y_{j}
$$

The $R_{c . J S M}$ model enhanced by the $A$ and $\boldsymbol{d}$ terms calculated according to (8)-(14) will be referred to as the "quick" SM-VWC model (QSM-VWC) and denoted as $R_{\text {S.QSM }-W W C}$. Note that QSM-VWC retains the properties of SM-VWC without compromising computational cost. In particular, parameters $A$ and $\boldsymbol{d}$ are obtained with an analytical formula based on the weighting factors dependent on the evaluation point $\boldsymbol{x}$ and the surrogate model evaluation does not require extraction of parameters based on the nonlinear minimization procedure as in the original SM-VWC method (cf. (3)). Also, the parameter $c$ in the QSM-VWC model can be easily optimized using crossvalidation [8], which is rather impracticable for SM-VWC because of the high computational cost of this process.

\section{EXAMPLES}

Here, we compare the standard SM modeling [5] (SMStandard), original SM-VWC [7], and the QSM-VWC method with respect to modeling accuracy as well as the computational complexity the surrogate model.

\section{A. Test Problem Description}

Problem 1: Microstrip right-angle bend [9]. The fine model, Fig. 1(a), is analyzed by Sonnet's em [10]. The coarse model is an equivalent circuit shown in Fig. 1(b). The design parameters are $\boldsymbol{x}=\left[\begin{array}{lll}W H & \varepsilon_{r}\end{array}\right]^{T}$. The response vector consists of reflection coefficient $\left|S_{11}\right|$ in the frequency range of 1 to $31 \mathrm{GHz}$. The reference point is $x^{0}$ $=\left[\begin{array}{lll}25 & 12 & 9\end{array}\right]^{T}$, and the region size is $\delta=\left[\begin{array}{lll}6 & 4\end{array}\right]^{T}$.

Problem 2: Second-order tapped-line microstrip filter [11] shown in Fig. 2. The fine model is simulated in FEKO [12]. The coarse model, Fig. 3, is the equivalent circuit model implemented in Agilent ADS [13]. The design parameters are $\boldsymbol{x}=\left[\begin{array}{ll}L_{1} & g\end{array}\right]^{T}$. The response vector consists of transmission coefficient $\left|S_{21}\right|$ in the frequency range 3 to $7 \mathrm{GHz}$. The reference point is $x^{0}=[6.977$ $0.060]^{T} \mathrm{~mm}$ and the region size is $\delta=\left[\begin{array}{ll}2 & 0.03\end{array}\right]^{T} \mathrm{~mm}$. 


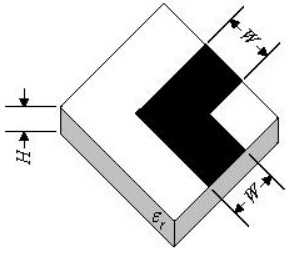

(a)

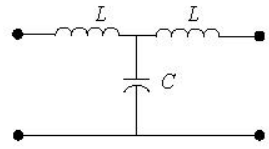

(b)
Fig.1. The microstrip right-angle bend [9]: the fine model (a) and the coarse model (b).

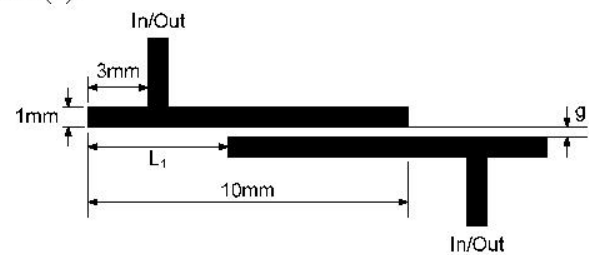

Fig. 2. Geometry of the second-order tapped-line microstrip filter [11].
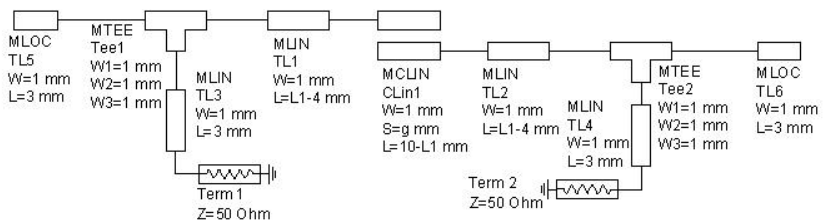

Fig. 3. Coarse model of the second-order tapped-line filter (Agilent ADS).

\section{B. Experimental Setup}

Table I shows details of the base sets used in our experiments. The standard SM model (1), SM-VWC, and QSM-VWC use the generic model (6) enhanced by frequency SM [5]. Accuracy was tested using 50 test points randomly distributed in the region of interest. The error measure used was the relative error $\| R_{f}(x)-$ $R_{s}(x)|| / \mid R_{f}(x) \|$ expressed in percent, where $R_{f}(x)$ and $R_{s}(x)$ denote the fine and the respective surrogate model response at a given test point $\boldsymbol{x}$ (here, $R_{s}(\boldsymbol{x})$ is either $R_{S . S M}(x), R_{S . S M-W W C}(x)$, or $\left.R_{S . Q S M-W W C}(x)\right)$. To reduce the evaluation time, the parameter extraction process in the SM-VWC model used a relaxed termination condition and exploited only those base points that satisfy $w_{k}(\boldsymbol{x})>0.01$.

\section{Results and Discussion}

Tables II and III show the modeling error and evaluation times for the considered surrogate models. Figs. 4 and 5 show the error plots, i.e., $\left|R_{f}(x)-R_{s}(x)\right|$ for the SM-Standard and QSM-VWC for Problems 1 and 2 respectively. Figs. 6 and 7 show the average error versus characteristic distance $\lambda$ for the considered models.

It is seen that the QSM-VWC model provides even better accuracy than the SM-VWC model and it is substantially faster than the latter. In particular, its evaluation time is of the same order of magnitude as the time for the SMStandard and about three orders of magnitude smaller than the time for the SM-VWC model. Therefore, the QSMVWC model can be used for optimization purposes.
TABLE I

Base Set Data For Test Problems 1 and 2

\begin{tabular}{|c|c|c|c|c|}
\hline $\begin{array}{c}\text { Test } \\
\text { Broblem }\end{array}$ & $\begin{array}{c}\text { Base } \\
\text { Set }\end{array}$ & Base Set Description & $\begin{array}{c}\text { Number of Base } \\
\text { Points }\end{array}$ & $\lambda$ \\
\hline \multirow{5}{*}{1} & $X_{B 1}$ & Uniform mesh of density 3 & 27 & 2.44 \\
\hline & $X_{B 2}$ & Uniform mesh of density 4 & 64 & 1.83 \\
\hline & $X_{B 3}$ & Uniform mesh of density 5 & 125 & 1.47 \\
\hline & $X_{B 4}$ & Uniform mesh of density 6 & 216 & 1.22 \\
\hline & $X_{B 5}$ & Uniform mesh of density 7 & 343 & 1.05 \\
\hline \multirow{5}{*}{2} & $X_{B 1}$ & Uniform mesh of density 3 & 9 & 0.68 \\
\hline & $X_{B 2}$ & Uniform mesh of density 4 & 16 & 0.51 \\
\hline & $X_{B 3}$ & Uniform mesh of density 5 & 25 & 0.41 \\
\hline & $X_{B 4}$ & Uniform mesh of density 7 & 49 & 0.29 \\
\hline & $X_{B 5}$ & Uniform mesh of density 9 & 81 & 0.23 \\
\hline
\end{tabular}

TABLE II

MODELING RESULTS FOR TEST PROBLEM 1

\begin{tabular}{ccccc}
\hline \hline Model & $\begin{array}{c}\text { Base } \\
\text { set }\end{array}$ & $\begin{array}{c}\text { Average } \\
\text { Error [\%] }\end{array}$ & $\begin{array}{c}\text { Maximum } \\
\text { Error [\%] }\end{array}$ & $\begin{array}{c}\text { Average Evaluation } \\
\text { Time }\end{array}{ }^{*}[\mathrm{~s}]$ \\
\hline SM-Standard & & 0.396 & 1.010 & 0.004 \\
SM-VWC & $X_{B 1}$ & 0.341 & 0.874 & 6.7 \\
QSM-VWC & & 0.190 & 0.640 & 0.004 \\
\hline SM-Standard & & 0.386 & 0.985 & 0.004 \\
SM-VWC & $X_{B 2}$ & 0.270 & 0.849 & 7.8 \\
QSM-VWC & & 0.123 & 0.344 & 0.006 \\
\hline SM-Standard & & 0.381 & 0.971 & 0.004 \\
SM-VWC & $X_{B 3}$ & 0.233 & 0.786 & 8.9 \\
QSM-VWC & & 0.092 & 0.385 & 0.012 \\
\hline SM-Standard & & 0.379 & 0.674 & 0.004 \\
SM-VWC & $X_{B 4}$ & 0.171 & 0.835 & 10.9 \\
QSM-VWC & & 0.064 & 0.204 & 0.019 \\
\hline SM-Standard & & 0.373 & 0.977 & 0.004 \\
SM-VWC & $X_{B 5}$ & 0.145 & 0.835 & 15.5 \\
QSM-VWC & & 0.036 & 0.130 & 0.030 \\
\hline \hline
\end{tabular}

All the models are run in the GUI-based SMF system [14]: the evaluation time includes the time required to run the auxiliary procedures including plotting; thus, the above values for SM-Standard and QSM-VWC are only estimates. They are larger than the actual evaluation times.

TABLE III

MODELING RESULTS FOR TEST PROBLEM 2

\begin{tabular}{ccccc}
\hline \hline Model & $\begin{array}{c}\text { Base } \\
\text { set }\end{array}$ & $\begin{array}{c}\text { Average } \\
\text { Error [\%] }\end{array}$ & $\begin{array}{c}\text { Maximum } \\
\text { Error [\%] }\end{array}$ & $\begin{array}{c}\text { Average Evaluation } \\
\text { Time }\end{array}$ \\
\hline SM-Standard & & 1.580 & 2.394 & 0.01 \\
SM-VWC & $X_{B 1}$ & 0.914 & 2.905 & 18.8 \\
QSM-VWC & & 0.627 & 1.784 & 0.012 \\
\hline SM-Standard & & 0.950 & 2.020 & 0.01 \\
SM-VWC & $X_{B 2}$ & 0.428 & 1.062 & 27.6 \\
QSM-VWC & & 0.213 & 0.535 & 0.014 \\
\hline SM-Standard & & 0.863 & 2.017 & 0.01 \\
SM-VWC & $X_{B 3}$ & 0.363 & 0.797 & 33.9 \\
QSM-VWC & & 0.157 & 0.471 & 0.016 \\
\hline SM-Standard & & 0.812 & 2.017 & 0.01 \\
SM-VWC & $X_{B 4}$ & 0.322 & 0.898 & 37.8 \\
QSM-VWC & & 0.106 & 0.340 & 0.020 \\
\hline SM-Standard & & 0.789 & 2.006 & 0.01 \\
SM-VWC & $X_{B 5}$ & 0.313 & 0.781 & 40.3 \\
QSM-VWC & & 0.079 & 0.254 & 0.026 \\
\hline \hline
\end{tabular}

*All the models are run in the GUI-based SMF system [14]: the evaluation time includes the time required to run the auxiliary procedures including plotting; thus, the above values for SM-Standard and QSM-VWC are only estimates. They are larger than the actual evaluation times. 


\section{CONCLUSIONS}

A new SM-based modeling methodology is presented which combines the accuracy of space mapping with variable weight coefficients and the computational efficiency and simplicity of linear regression models. The numerical results show that our technique is competitive with both the standard SM approach and SM-VWC.

\section{ACKNOWLEDGEMENTS}

This work was supported in part by the Natural Sciences and Engineering Research Council of Canada under Grants RGPIN7239-06, STPGP336760-06. The authors thank Sonnet Software, Inc., Syracuse, NY, for $\boldsymbol{e m}^{\mathrm{TM}}$, and Agilent Technologies, Santa Rosa, CA, for ADS.

\section{REFERENCES}

[1] J.W. Bandler, Q.S. Cheng, S.A. Dakroury, A.S. Mohamed, M.H. Bakr, K. Madsen, and J. Søndergaard, "Space mapping: the state of the art," IEEE Trans. Microwave Theory Tech., vol. 52, no. 1, pp. 337-361, Jan. 2004.

[2] S. Koziel, J.W. Bandler, and K. Madsen, "A space mapping framework for engineering optimization: theory and implementation," IEEE Trans. Microwave Theory Tech., vol. 54, no. 10 , pp. $3721-3730$, Oct. 2006 .

[3] D. Echeverria and P.W. Hemker, "Space mapping and defect correction," CMAM The Int. Mathematical Journal Computational Methods in Applied Mathematics, vol. 5, no. 2, pp. 107-136, 2005.

[4] J.E. Rayas-Sánchez and V. Gutiérrez-Ayala, "EM-based Monte Carlo analysis and yield prediction of microwave circuits using linear-input neural-output space mapping," IEEE Trans. Microwave Theory Tech., vol. 54, no. 12, pp. 4528-4537, Dec. 2006.

[5] S. Koziel, J.W. Bandler, A.S. Mohamed, and K. Madsen, "Enhanced surrogate models for statistical design exploiting space mapping technology," IEEE MTT-S Int. Microwave Symp. Dig., Long Beach, CA, June 2005, pp. 1609-1612.

[6] L. Zhang, J.J. Xu, M. Yagoub, R.T. Ding, and Q.J. Zhang, "Neurospace mapping technique for nonlinear device modeling and large signal simulation," IEEE MTT-S Int. Microwave Symp. Dig., Philadelphia, PA, June 2003, pp. 173-176.

[7] S. Koziel, J.W. Bandler, and K. Madsen, "Theoretical justification of space-mapping-based modeling utilizing a data base and ondemand parameter extraction," IEEE Trans. Microwave Theory Tech., vol. 54, no. 12, pp. 4316-4322, Dec. 2006.

[8] N.V. Queipo, R.T. Haftka, W. Shyy, T. Goel, R. Vaidynathan, and P.K. Tucker, "Surrogate-based analysis and optimization," Progress in Aerospace Sciences, vol. 41, no. 1, pp. 1-28, Jan. 2005.

[9] J.W. Bandler, N. Georgieva, M.A. Ismail, J.E. Rayas-Sánchez, and Q. J. Zhang, "A generalized space mapping tableau approach to device modeling," IEEE Trans. Microwave Theory Tech., vol. 49, no. 1 , pp. 67-79, Jan. 2001.

[10] $\mathbf{e m}^{\mathrm{TM}}$, ver. 11.54, Sonnet Software, Inc., 100 Elwood Davis Road, North Syracuse, NY 13212, USA, 2007.

[11] A. Manchec, C. Quendo, J.-F. Favennec, E. Rius, and C. Person, "Synthesis of capacitive-coupled dual-behavior resonator (CCDBR) filters," IEEE Trans. Microwave Theory Tech, vol. 54, no. 6, pp. 2346-2355, June 2006.
[12] FEKO User's Manual, Suite 5.3, July 2007, EM Software \& Systems-S.A. (Pty) Ltd, 32 Techno Lane, Technopark, Stellenbosch, 7600, South Africa, http://www feko.info.

[13] Agilent ADS, Version 2003C, Agilent Technologies, 1400 Fountaingrove Parkway, Santa Rosa, CA 95403-1799, 2003.

[14] S. Koziel and J.W. Bandler, "SMF: a user-friendly software engine for space-mapping-based engineering design optimization," Int. Symp. Signals, Systems and Electronics, URSI ISSSE 2007, Montreal, Canada, July 2007, pp. 157-160.

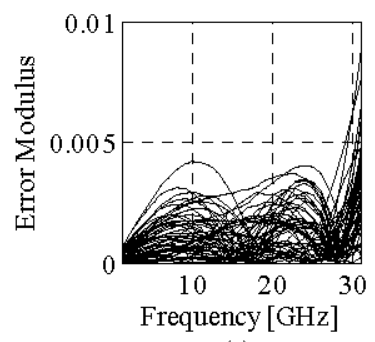

(a)

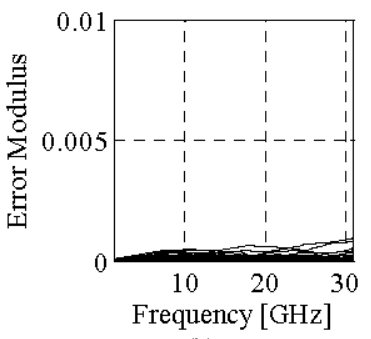

(b)
Fig.4. Test problem 1: error plots for the SM-Standard (a) and QSMVWC (b) surrogate models with base set $X_{B 5}$ (50 test points).

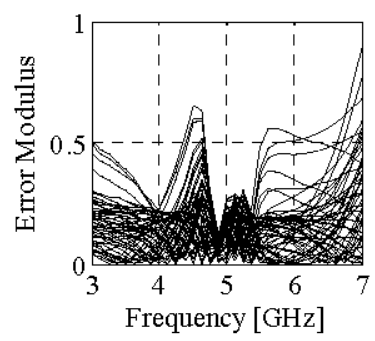

(a)

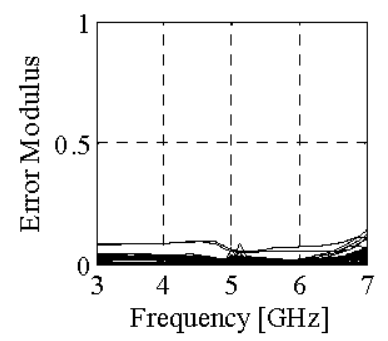

(b)
Fig.5. Test problem 2: error plots for the SM-Standard (a) and QSMVWC (b) surrogate models with base set $X_{B 3}$ ( 30 test points).

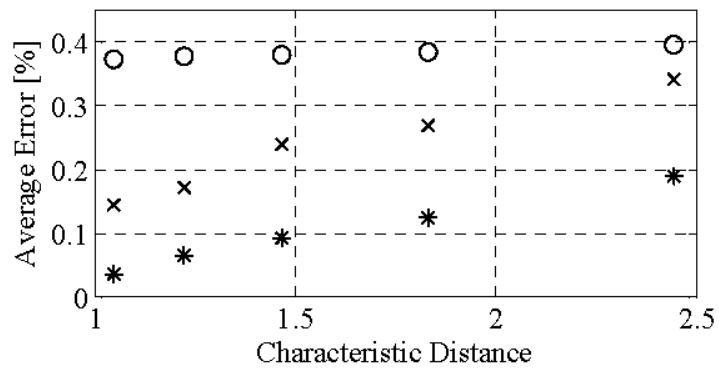

Fig.6. Test problem 1: average modeling error versus $\lambda$ : SM-Standard(o), SM-VWC $(\times)$, and QSM-VWC (*).

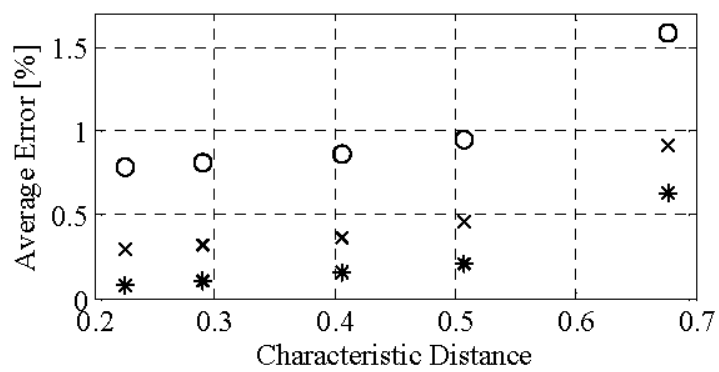

Fig.7. Test problem 2: average modeling error versus $\lambda$ : SM-Standard(o), SM-VWC $(\times)$, and QSM-VWC $(*)$ 